\title{
Enhanced $n$-Doping Efficiency of a Naphthalenediimide-Based Copolymer through Polar Side Chains for Organic Thermoelectrics
}

David Kiefer, ${ }^{\dagger}$ Alexander Giovannitti, ${ }^{\ddagger}$ Hengda Sun, ${ }^{\S}$ Till Biskup, $^{\perp} \odot$ Anna Hofmann, ${ }^{\dagger}$ Marten Koopmans, Camila Cendra, ${ }^{\#}$ Stefan Weber, ${ }^{\perp}$ L. Jan Anton Koster, ${ }^{\circledR}{ }^{\circ}$ Eva Olsson, ${ }^{\nabla}$ Jonathan Rivnay, ${ }^{\bigcirc}$ Simone Fabiano, ${ }^{\S}$ Iain McCulloch, ${ }^{\ddagger}$, and Christian Müller* ${ }^{*} \dagger$ (1)

${ }^{\dagger}$ Department of Chemistry and Chemical Engineering, Chalmers University of Technology, 41296 Göteborg, Sweden

${ }^{\ddagger}$ Department of Chemistry and Centre for Plastic Electronics, Imperial College London, London SW7 2AZ, United Kingdom

${ }^{\S}$ Laboratory of Organic Electronics, Department of Science and Technology, Linköping University, 60174 Norrköping, Sweden

${ }^{\perp}$ Institut für Physikalische Chemie, Albert-Ludwigs-Universität Freiburg, 79104 Freiburg, Germany

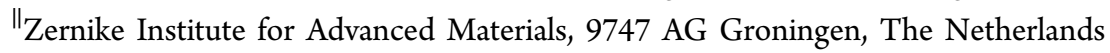

\# Department of Materials Science and Engineering, Stanford University, Stanford, California 94304, United States

${ }^{\nabla}$ Department of Physics, Chalmers University of Technology, 41296 Göteborg, Sweden

ODepartment of Biomedical Engineering, Northwestern University, Evanston, Illinois 60035, United States

SPERC, King Abdullah University of Science and Technology, Thuwal 23955-6900, Saudi Arabia

\section{Supporting Information}

ABSTRACT: $\mathrm{N}$-doping of conjugated polymers either requires a high dopant fraction or yields a low electrical conductivity because of their poor compatibility with molecular dopants. We explore ndoping of the polar naphthalenediimide-bithiophene copolymer p(gNDI-gT2) that carries oligoethylene glycol-based side chains and show that the polymer displays superior miscibility with the benzimidazole-dimethylbenzenamine-based $n$-dopant $\mathrm{N}$-DMBI. The good compatibility of $\mathrm{p}$ (gNDI-gT2) and N-DMBI results in a relatively high doping efficiency of $13 \%$ for $n$-dopants, which leads to a high electrical conductivity of more than $10^{-1} \mathrm{~S} \mathrm{~cm}^{-1}$ for a dopant concentration of only $10 \mathrm{~mol} \%$ when measured in an inert atmosphere. We find that the doped polymer is able to maintain its electrical conductivity for about 20 min when exposed to air and recovers rapidly when returned to a nitrogen atmosphere. Overall, solution coprocessing of $\mathrm{p}(\mathrm{gNDI}-\mathrm{gT2})$ and N-DMBI results in a larger thermoelectric power factor of up to $0.4 \mu \mathrm{W} \mathrm{K} \mathrm{K}^{-2}$ compared to other NDI-based polymers.

$\mathrm{D}$ oping of organic semiconductors is essential for the optimization of a number of electronic components, ranging from the hole and electron blocking layers used in organic solar cells ${ }^{1-3}$ and organic light-emitting diodes (OLEDs) $)^{1,4,5}$ to trap filling in organic field-effect transistors (OFETs) ${ }^{6-8}$ and the legs of thermoelectric generators. ${ }^{9,10}$ For many of these applications, conjugated polymers are particularly intriguing because they permit one to adjust the rheological properties of processing solutions and the mechanical properties of the final (flexible) thin film architectures. Doping can be achieved through electron transfer between the semiconductor and a molecular dopant via a redox

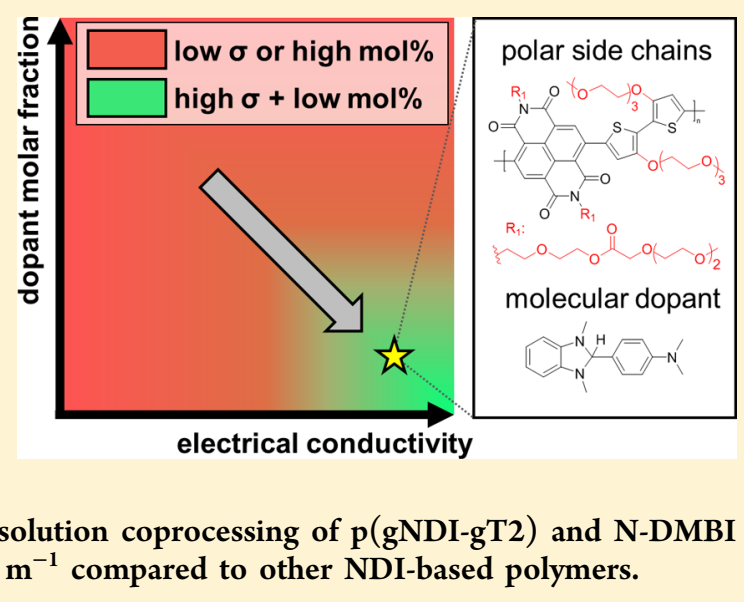

reaction. Alternatively, a proton/hydride $\left(\mathrm{H}^{+} / \mathrm{H}^{-}\right)$can be transferred from an acid/base to the semiconductor. ${ }^{11}$ In the case of p-doping, positive charge carriers are introduced, whereas $n$-doping refers to the addition of extra electrons to the conjugated system. It is desirable that each dopant molecule that is added to the semiconductor material introduces as many charges as possible. Therefore, the presence of unreacted dopant should be avoided in order to maximize the amount of

Received: November 19, 2017

Accepted: December 27, 2017 
conducting material. Moreover, the presence of excess dopant has the tendency to disrupt the nanostructure of the neat semiconductor, which can negatively impact charge transport. ${ }^{10,12-14}$ Hence, it is critical that the doping efficiency, i.e., the fraction of dopants that ultimately create a charge on the organic semiconductor, be as high as possible. ${ }^{15}$

To realize thermoelectric generators, both $\mathrm{p}$ - and n-type materials are needed. They should display a high figure of merit $Z T=\alpha^{2} \sigma \cdot T / \kappa$, where $\alpha$ is the Seebeck coefficient, $\sigma$ the electrical conductivity, $T$ the absolute temperature, and $\kappa$ the thermal conductivity. If the thermal conductivity, which is challenging to measure for thin film architectures, is unknown, the power factor $\alpha^{2} \sigma$ is instead used to compare the thermoelectric efficacy of different materials. P-doping of conjugated polymers is now well established ${ }^{6,16,17}$ and can be carried out with high efficiency and with high environmental stability, leading to a thermoelectric power factor of at least 100 $\mu \mathrm{W} \mathrm{m}{ }^{-1} \mathrm{~K}^{-2} \cdot{ }^{18-20}$ In contrast, $\mathrm{n}$-doping continues to pose a formidable challenge because of very low doping efficiencies as well as poor stability of the doped state. ${ }^{21}$ The most widely studied classes of n-type materials include naphthalenediimide (NDI)-based copolymers, ${ }^{14,22-29}$ diketopyrrolopyrrole (DPP)based polymers, ${ }^{30-32}$ as well as fullerenes and their derivatives. ${ }^{3,8,33-45}$ We have compiled data from the literature to compare the dopant fractions that are required to achieve the maximum conductivity $\sigma_{\max }$ through $\mathrm{n}$-doping of various semiconductors (Figure 1; Supporting Information Table S1). It is evident that $\mathrm{n}$-doping of NDI-based polymers is limited by a too low doping efficiency. The result is either a low maximum electrical conductivity of less than $10^{-2} \mathrm{~S} \mathrm{~cm}^{-1}$ at low dopant fractions (Figure 1a; bottom left) or the need for a large dopant fraction of more than $30 \mathrm{~mol} \%$ to achieve a higher electrical conductivity (Figure 1a; top right). For example, Schlitz et al. investigated $\mathrm{n}$-doping of the high-mobility naphthalenediimide-bithiophene copolymer $\mathrm{p}(\mathrm{NDI} 2 \mathrm{OD}-\mathrm{T} 2)^{46}$ with the commonly used n-dopant N-DMBI (see Figure 2 for the chemical structure) and reached an electrical conductivity of about $10^{-3} \mathrm{~S} \mathrm{~cm}^{-1}$ at a dopant fraction of $9 \mathrm{~mol} \%{ }^{14}$ The insolubility of N-DMBI in the host polymer leading to segregation of the dopant was noted to be a limiting effect for the electrical properties. Naab et al. studied doping of several NDI-based polymers with a dimer version of DMBI and found that a dopant fraction of up to $43 \mathrm{~mol} \%$ was required to maximize the electrical conductivity, ${ }^{26}$ despite a higher doping efficiency, because each dimer can create two charges. ${ }^{42}$

One emerging tool to increase the doping efficiency is the replacement of nonpolar alkyl side chains with more polar oligoethylene glycol side chains, which enhances the compatibility of semiconductor/dopant pairs. ${ }^{17} \mathrm{Li}$ et al. have observed that the common p-dopant F4TCNQ more readily diffuses into a polythiophene that carries oligoethylene glycol side chains as well as a sulfonate group, as compared to poly(3-hexylthiophene) (P3HT), which indicates that polar side chains can improve dopant miscibility. ${ }^{49}$ As a result, polar side chains can lead to complete p-doping efficiency of polythiophenes by F4TCNQ, resulting in both a $\sigma_{\max } \approx 100 \mathrm{~S} \mathrm{~cm}^{-1}$ for a low dopant fraction of $10 \mathrm{~mol} \%$ as well as enhanced thermal stability. ${ }^{13}$ Likewise, fullerenes that carry oligoethylene glycol side chains feature enhanced compatibility with N-DMBI and therefore a high doping efficiency of about $18 \%$, which yielded a maximum conductivity of about $2 \mathrm{~S} \mathrm{~cm}^{-1}$ and power factor of up to $19 \mu \mathrm{W} \mathrm{m} \mathrm{W}^{-1} \mathrm{~K}^{-2} \cdot 39,43$ a

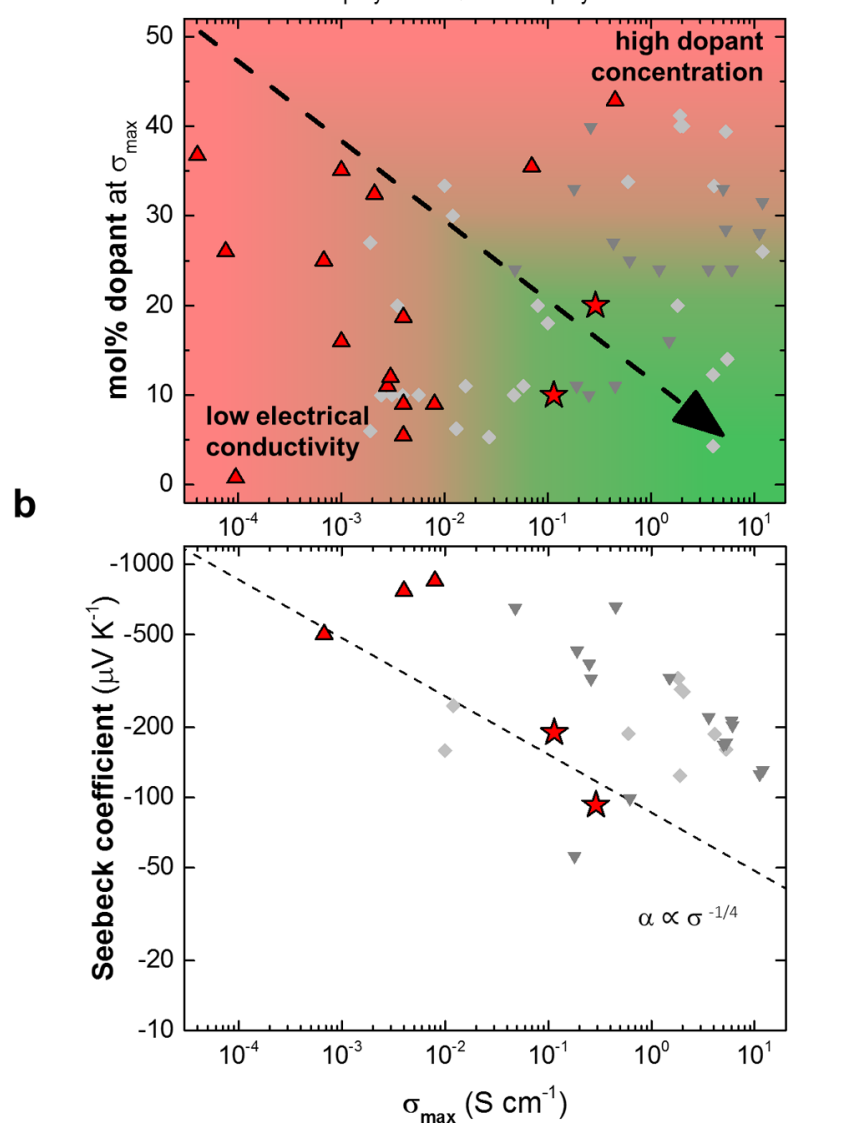

Figure 1. (a) Literature values of the molar dopant fraction required to reach the maximum electrical conductivity $\left(\sigma_{\max }\right)$ for $\mathbf{n}$ doped NDI-based polymers $(\boldsymbol{\Delta}){ }^{14,22-24,26,27,29}$ other (e.g DPP- or NTDI-based) polymers $(\boldsymbol{\nabla}),{ }^{30-32,47,48}$ fullerene derivatives $(\downarrow),{ }^{8,34,36-43,45}$ and p(gNDI-gT2) ( $\star$, this work); (b) corresponding Seebeck coefficient $(\alpha)$ at maximum electrical conductivity; empirical relation $\alpha \propto \sigma^{-1 / 4}$. $^{10}$

In this work, we explore n-doping of the naphthalenediimide-bithiophene copolymer p(gNDI-gT2) (for details on synthesis and characterization, see the Supporting Information and Figures S1 and S2), a structural analogue of $\mathrm{p}$ (NDI2ODT2) with polar oligoethylene glycol-containing side chains on both the NDI acceptor and the bithiophene donor unit, which has proven to be a promising material for organic electrochemical transistors (OECTs). ${ }^{50-52}$ We anticipate that the structural alteration from nonpolar alkyl side chains to more polar oligoethylene glycol side chains will aid doping of the polymer backbone through enhanced dopant miscibility. We chose to investigate n-doping with N-DMBI, which is thought to donate a hydride $\left(\mathrm{H}^{-}\right),{ }^{27,42,53,54}$ and found that our best results in terms of doping efficiency and maximum conductivity are superior to previous results that have been reported for other n-type polymers (Figure 1a, bottom right, green).

In a first set of experiments, we recorded UV/vis spectra of $\mathrm{p}$ (gNDI-gT2) solutions (Figure 3a) and films (Figure 3b) before and after addition of N-DMBI. The thin film spectrum of the pristine polymer consists of a peak at around $\sim 440 \mathrm{~nm}$ and a broad spectral feature between 600 and $1500 \mathrm{~nm}$, which we attribute to the $\pi-\pi^{*}$ transition and a strong intramolecular charge transfer complex as a consequence of strong donoracceptor interactions. ${ }^{50,55}$ Because Giovannitti et al. observed 
p(gNDI-gT2)<smiles>[R1]N1C(=O)c2cc(-c3cc(OCCOC)c(-c4sc(F)cc4OCCOC)s3)c3c4c(cc(C)c(c24)C1=O)C(=O)N([R1])C3=O</smiles><smiles>[R]CCOCCOC(=O)COCCOC</smiles>

b N-DMBI<smiles>CN(C)c1ccc(C2(C)N(C)c3ccccc3N2C)cc1</smiles>

Figure 2. Chemical structures of (a) $\mathrm{p}(\mathrm{gNDI}-\mathrm{gT} 2)^{50}$ and (b) the molecular dopant N-DMBI. very little variation of the higher-energy absorption peak upon electrochemical doping, we chose to normalize all spectra to this peak for comparison. We note that for slight doping with $10 \mathrm{~mol} \% \mathrm{~N}$-DMBI the low-energy absorption peak slightly increases. Instead, upon additional doping, the broad spectral feature at higher wavelengths diminishes, while the absorption at around $600 \mathrm{~nm}$ increases relative to the peak at $440 \mathrm{~nm}$ after doping with N-DMBI. The latter trend is in full agreement with the study by Giovannitti et al. and previous literature on $\mathrm{n}$ doping. ${ }^{26,28,50}$ Doping results in a gradual red shift of the lowenergy absorption peak from $1016 \mathrm{~nm}$ for the pristine polymer to $1040 \mathrm{~nm}$ for $\mathrm{p}$ (gNDI-gT2) doped with $50 \mathrm{~mol} \% \mathrm{~N}$-DMBI. We tentatively assign this red shift as well as the slight increase in absorption upon doping with $10 \mathrm{~mol} \% \mathrm{~N}$-DMBI to planarization of the polymer backbone. Interestingly, we note that the addition of N-DMBI has seemingly no effect on the solution spectra of dissolved $\mathrm{p}$ (gNDI-gT2). Thus, we conclude that doping of the polymer is likely to occur during the film formation step upon solvent removal.

To obtain an estimate of the charge carrier density $(n)$, we used the change in the activation energy of the conductivity upon doping. The estimation is based on the extended Gaussian disorder model (EGDM) ${ }^{56}$ as reported by Liu et al. ${ }^{39}$ The model yields a general relationship of the chargecarrier density and $E_{\mathrm{a}} / E_{0}$, where $E_{\mathrm{a}}$ and $E_{0}$ are the activation energies at a certain doping fraction and at a low carrier density

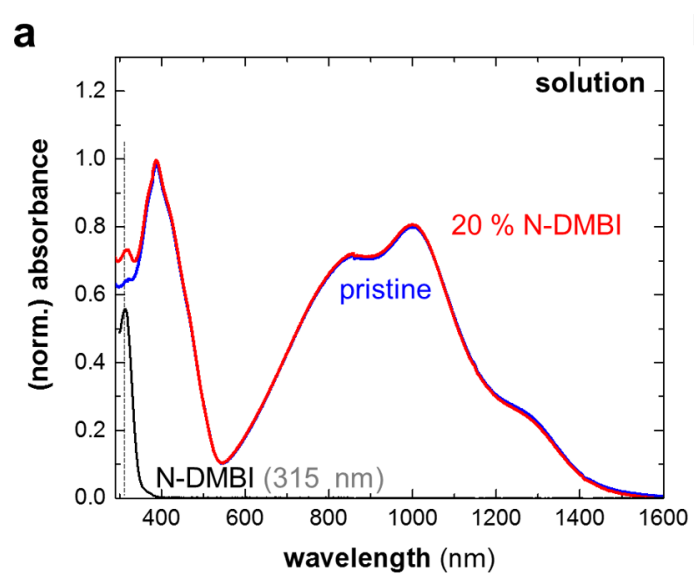

b
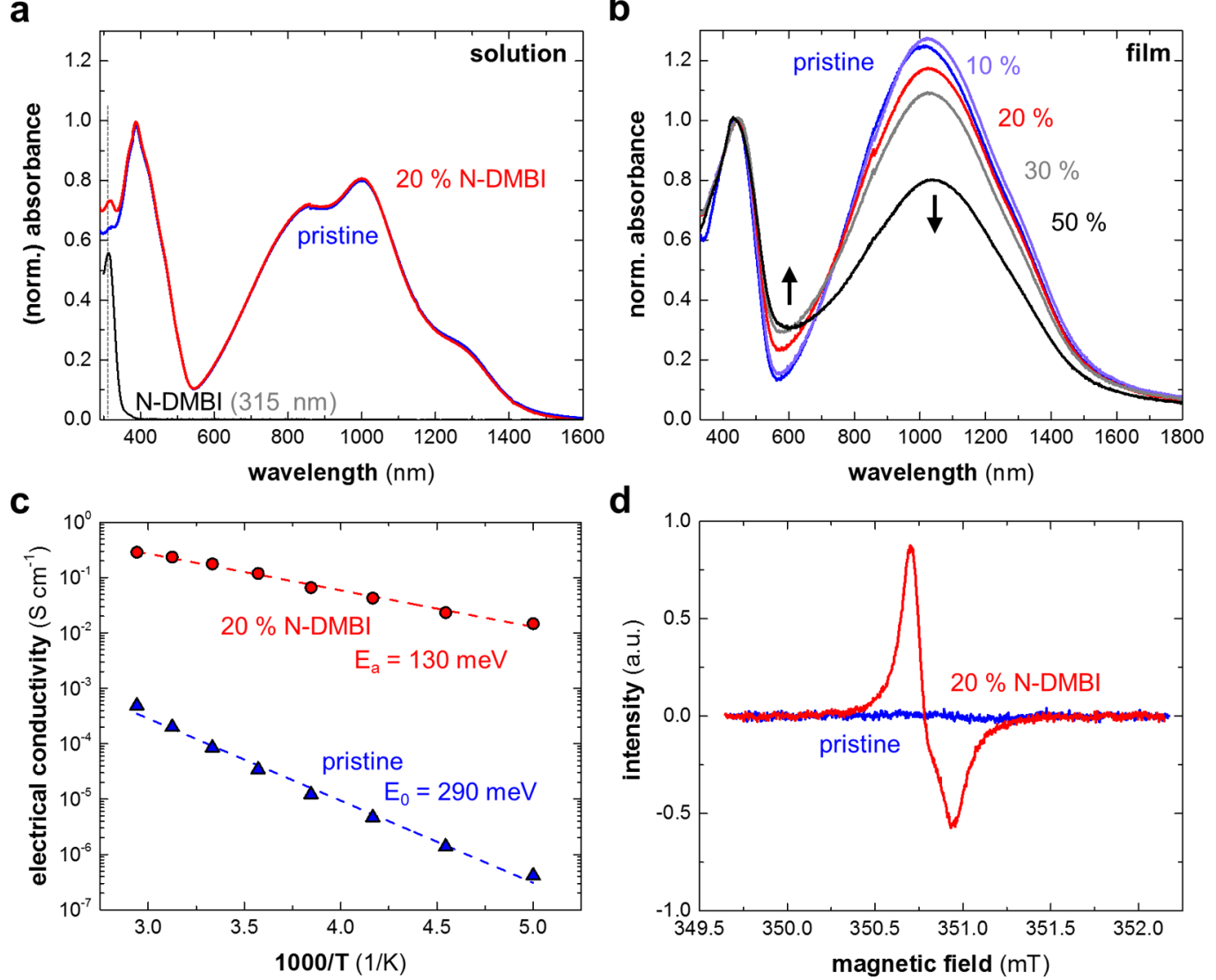

Figure 3. (a) Solution absorbance spectra of pristine p(gNDI-gT2), p(gNDI-gT2) + 20 mol \% N-DMBI (note that the spectral feature at 315 $\mathrm{nm}$ is due to neat N-DMBI), and neat N-DMBI in chloroform; (b) normalized absorbance spectra of pristine and N-DMBI-doped $\mathrm{p}$ (gNDIgT2) films (10, 20, 30, and $50 \mathrm{~mol} \% \mathrm{~N}-\mathrm{DMBI})$; (c) Arrhenius plots of variable-temperature conductivity measurements (dashed lines are fits to the Arrhenius equation, yielding the activation energies $E_{0}$ and $E_{\mathrm{a}}$ ); and (d) electron paramagnetic resonance (EPR) spectra of pristine and N-DMBI-doped p(gNDI-gT2) films. 

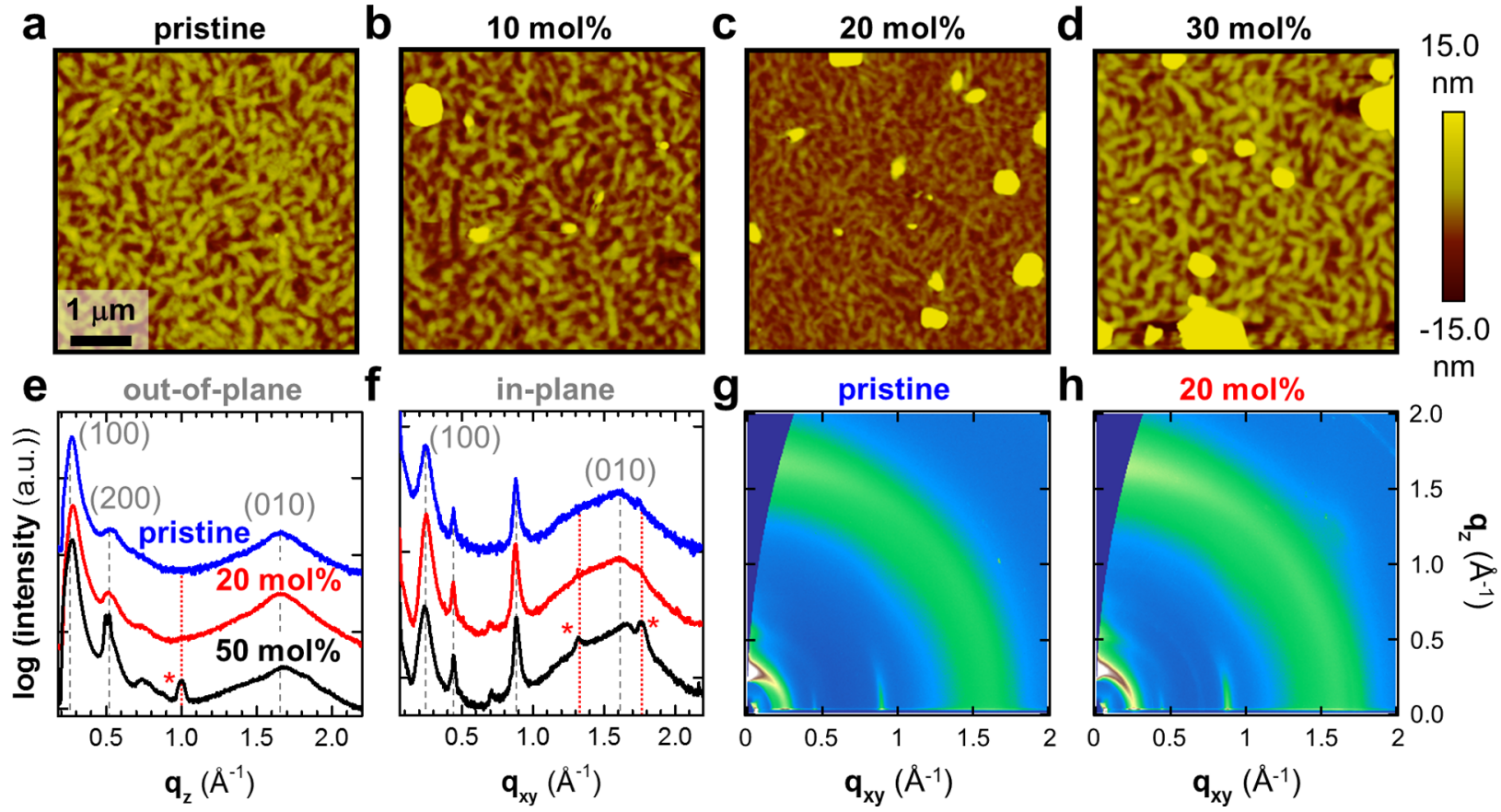

Figure 4. Atomic force microscopy (AFM) height images of (a) pristine, and N-DMBI-doped p(gNDI-gT2): (b) 10, (c) 20, and (d) 30 mol \% N-DMBI. X-ray diffractograms of pristine and doped p(gNDI-gT2) obtained by integration along the (e) out-of-plane $\left(q_{z}\right)$ and (f) in-plane $\left(q_{x y}\right)$ direction. Scattering from lamellar and $\pi$-stacking is indicated with $(h 00)$ and $(0 k 0)$; scattering marked with an asterisk $(*)$ is associated with the neat dopant. 2D grazing-incidence wide-angle X-ray scattering images of (g) pristine p(gNDI-gT2) and (h) the polymer doped with 20 mol \% N-DMBI.

(pristine material) for a specific disorder parameter, respectively. The activation energies of pristine and doped $\mathrm{p}$ (gNDIgT2) were extracted from variable-temperature electrical conductivity measurements by fitting an Arrhenius temperature dependence (Figure 3c)

$$
\sigma=\sigma_{0} \mathrm{e}^{-E_{\mathrm{a}} /\left(k_{\mathrm{B}} T\right)}
$$

where $E_{\mathrm{a}}$ is the activation energy, $k_{\mathrm{b}}$ the Boltzmann constant, and $\sigma_{0}$ a pre-exponential factor that does not influence the activation energy. We obtained activation energies of $E_{0}=290$ $\mathrm{meV}$ and $E_{\mathrm{a}}=130 \mathrm{meV}$ for the pristine polymer and a sample doped with $20 \mathrm{~mol} \% \mathrm{~N}$-DMBI, respectively. We extracted a disorder parameter of $90 \mathrm{meV}$ and hence estimated a charge carrier density of $1.5 \times 10^{19} \mathrm{~cm}^{-3}$, assuming an average hopping distance for conjugated polymers of $1 \mathrm{~nm}^{57,58}$ and an overall density of states of $10^{21} \mathrm{~cm}^{-3}$ (see Supporting Information Figure S3 for details). Note that we can produce good fits for nearest-neighbor hopping as well as 1-, 2-, and 3D variable range hopping, which prevents us from determining the transport mode based on our data (see Supporting Information Figure S4).

To corroborate the estimated charge carrier density of $\mathrm{N}$ DMBI doped p(gNDI-gT2), we employed electron paramagnetic resonance (EPR) spectroscopy (Figure 3d). In the case of (negative) polarons as predominant charge carrier species, the electron spin density acquired by measurement against a known reference sample is directly equivalent to the charge carrier concentration. The lack of an EPR signal for the pristine polymer indicates that the number of unpaired electrons is low. In contrast, for a sample doped with $20 \mathrm{~mol}$ $\% \mathrm{~N}-\mathrm{DMBI}$, we readily observe an EPR signal, indicating that $\mathrm{n}$-doping of the polymer has indeed taken place. Quantification of the spectra yields a spin density of $\sim 1.0 \times 10^{19} \mathrm{~cm}^{-3}( \pm 0.3 \times$
$10^{19} \mathrm{~cm}^{-3}$ ). This value is consistent with our estimate for the charge carrier density from the EGDM model, which indicates that polarons are the predominant type of charge carriers because bipolarons would not give rise to an EPR signal. We explain the absence of an EPR signal for the neat polymer despite considerable background doping (cf. discussion below), with the 50 times lower conductivity and hence polaron concentration, which means that our measurement is not sensitive enough.

Comparison of the number of charge carriers $n$ and the total number of N-DMBI molecules $n_{\mathrm{N}-\mathrm{DMBI}}$ allows us to estimate the doping efficiency, i.e., the ratio $n / n_{\mathrm{N}-\mathrm{DMBI}}$. A dopant concentration of $20 \mathrm{~mol} \%$ translates into $1.3 \times 10^{20} \mathrm{~cm}^{-3} \mathrm{~N}$ DMBI molecules, assuming a density of $1 \mathrm{~g} \mathrm{~cm}^{-3}$. Hence, we estimate an approximate doping efficiency of about $13 \%$ for $\mathrm{p}$ (gNDI-gT2) doped with $20 \mathrm{~mol} \% \mathrm{~N}-\mathrm{DMBI}$. In comparison, Schlitz et al. have deduced a more than 10-times lower NDMBI doping efficiency of only $1 \%$ for the nonpolar $\mathrm{p}$ (NDI2OD-T2). ${ }^{14}$ In analogy to several studies of polythiophenes ${ }^{13,49}$ and fullerenes ${ }^{39,43}$ decorated with more polar oligoethylene glycol moieties, we attribute the higher doping efficiency of N-DMBI-doped p(gNDI-gT2) to enhanced miscibility of the polymer/dopant pair.

The low doping efficiency of polymers such as $\mathrm{p}$ (NDI2OD$\mathrm{T} 2)$ results in the formation of numerous N-DMBI aggregates on the film surface, which become clearly visible for a doping fraction as low as $9 \mathrm{~mol} \%{ }^{14}$ We therefore anticipate that the superior doping efficiency of $\mathrm{p}(\mathrm{gNDI}-\mathrm{gT} 2)$ reduces the tendency for N-DMBI aggregation. We employed atomic force microscopy (AFM) and scanning electron microscopy (SEM) to study the surface topography of $\mathrm{p}$ (gNDI-gT2) thin films (Figure 4a-d; Supporting Information Figures S5-S7). Both AFM and SEM images indicate formation of dopant aggregates on the surface of the blend films that increase in 
quantity and size with an increasing amount of N-DMBI. The surface roughness (Supporting Information Figure S8) changes only slightly from $2 \mathrm{~nm}$ for the pristine film to $6 \mathrm{~nm}$ after up to $20 \mathrm{~mol} \% \mathrm{~N}-\mathrm{DMBI}$ is added but increases sharply for $30 \mathrm{~mol} \%$ and more. Intriguingly, the surface roughness in the regions between the aggregates is not significantly affected by doping, even at higher doping fractions, which suggests that the nanostructure of the pristine polymer is largely maintained.

To further elucidate the effect of the dopant on the nanostructure of the polymer, we obtained a series of scattering diffractograms in the out-of-plane and in-plane directions (Figure 4e,f) through integration of grazing-incidence wideangle scattering (GIWAXS) images of pristine and heavily doped $\mathrm{p}(\mathrm{gNDI}-\mathrm{gT} 2)$ (Figure 4g,h; Supporting Information Figure S10). The pristine polymer features distinct scattering peaks from lamellar stacking at $q_{100} \approx 0.27 \AA^{-1}$ and $q_{200} \approx 0.54$ $\AA^{-1}$ and from $\pi$-stacking at $q_{010} \approx 1.6 \AA^{-1}$. Further, in the inplane scan, two additional peaks are present at $q_{x y} \approx 0.45 \AA^{-1}$ and $q_{x y} \approx 0.9 \AA^{-1}$. We assign these peaks to the repeat distance along the backbone and argue that, similar to $\mathrm{p}$ (NDI2ODT2), ${ }^{5-62}$ two polymorphs are present. The diffraction peaks that we observe for pristine $\mathrm{p}$ (gNDI-gT2) are not altered upon doping with $20 \mathrm{~mol} \% \mathrm{~N}-\mathrm{DMBI}$. Addition of $50 \mathrm{~mol} \%$ dopant results in the appearance of a new out-of-plane scattering peak at $q_{z} \approx 1 \AA^{-1}$ and in-plane at $q_{x y} \approx 1.3 \AA^{-1}$ as well as $q_{x y} \approx 1.75$ $\AA^{-1}$, which we explain with the presence of unreacted excess dopant. Further, annealing of the films does not alter the diffraction from the polymer but results in a slight shift of the peaks associated with excess N-DMBI, as well as a decrease in scattering intensity (Supporting Information Figure S11). We conclude that significant segregation only takes place for a dopant concentration above $20 \mathrm{~mol} \%$. Note that a few isolated aggregates are already visible in the AFM images of $\mathrm{p}$ (gNDIgT2) doped with $20 \mathrm{~mol} \% \mathrm{~N}-\mathrm{DMBI}$, which are weakly visible in the GIWAXS measurements. Comparison with the nonpolar $\mathrm{p}$ (NDI2OD-T2) (cf. study by Schlitz et al. ${ }^{14}$ ) indicates that the polar oligoethylene glycol side chains largely suppress N-DMBI aggregation up to a concentration of about $20 \mathrm{~mol} \%$, which is consistent with our picture of enhanced polymer/dopant miscibility.

In a further set of experiments, we characterized the electrical properties of $\mathrm{p}(\mathrm{gNDI}-\mathrm{gT} 2) \approx 60 \mathrm{~nm}$ thin films doped with various amounts of N-DMBI (Figure 5). The pristine polymer features an electrical conductivity of $6 \times 10^{-3} \mathrm{~S} \mathrm{~cm}^{-1}$, which arises due to background doping. In a first regime up to $20 \mathrm{~mol}$ $\%$, the addition of N-DMBI is concomitant with an increase in electrical conductivity. We reach a value above $10^{-1} \mathrm{~S} \mathrm{~cm}^{-1}$, which is more than 2 orders of magnitude higher than $\mathrm{p}$ (NDI2OD-T2) doped with N-DMBI (Supporting Information Figure S12a) due to the here-reported higher doping efficiency in the case of $\mathrm{p}$ (gNDI-gT2). At the same time, for a dopant concentration up to $20 \mathrm{~mol} \%$, the Seebeck coefficient decreases from 359 to $93 \mu \mathrm{V} \mathrm{K} \mathrm{K}^{-1}$. Upon further doping, we observe a substantial drop of the electrical conductivity by nearly 2 orders of magnitude. In contrast, in this second regime, the Seebeck coefficient only slightly decreases to, e.g., $70 \mu \mathrm{V}$ $\mathrm{K}^{-1}$ for $30 \mathrm{~mol} \% \mathrm{~N}-\mathrm{DMBI}$, indicating that the number of mobile charge carriers is not strongly enhanced upon further addition of N-DMBI. We rationalize this behavior with gradual disruption of the polymer nanostructure by excess unreacted dopant, which coincides with the appearance of N-DMBI aggregates (cf. Figure 4). a
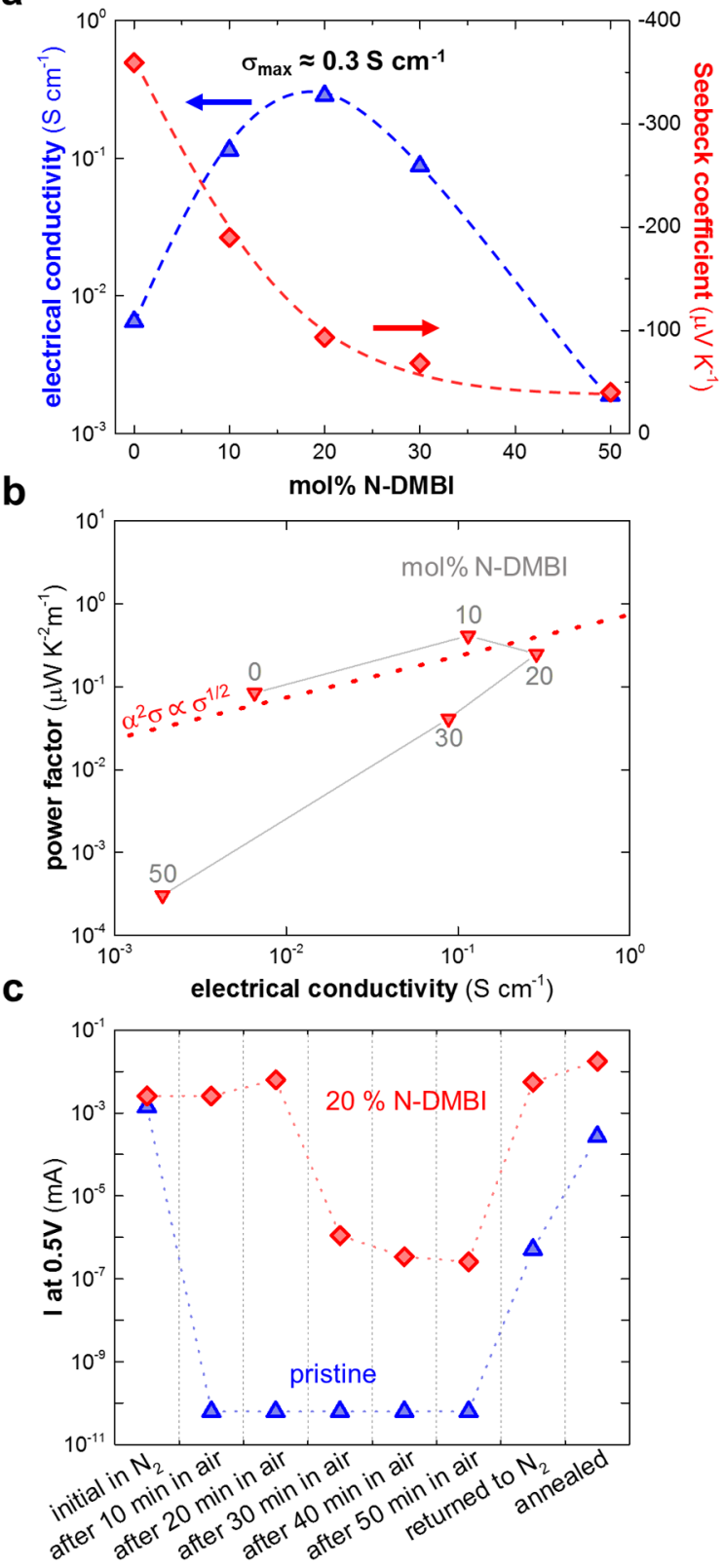

Figure 5. (a) Electrical conductivity $(\sigma)$ and Seebeck coefficient $(\alpha)$; dashed lines are a guide to the eye. (b) Thermoelectric power factor $\left(\alpha^{2} \sigma\right)$ as a function of the electrical conductivity at various dopant fractions; the dashed line represents the empirical relation $\alpha^{2} \sigma \propto \sigma^{1 / 2}{ }^{10}$ (c) Air stability of pristine and N-DMBI-doped $\mathrm{p}$ (gNDI-gT2): the current at $0.5 \mathrm{~V}$ was extracted from $I-V$ curves recorded in nitrogen, in air, and finally again in nitrogen; note that the nonohmic behavior of several samples prevented us from extracting the electrical conductivity. A contact geometry with a channel length of $1000 \mu \mathrm{m}$ and a channel width of $30 \mu \mathrm{m}$ was used for air stability measurements of doped samples, which resulted in similar currents for the pristine and doped sample.

We chose to compare the thermoelectric performance of $\mathrm{N}$ DMBI-doped $\mathrm{p}$ (gNDI-gT2) with the empirical correlation that Glaudell et al. have proposed for the thermoelectric power factor of not mobility-limited p-doped semiconductors: $\alpha^{2} \sigma \propto$ $\sigma^{1 / 2} \cdot{ }^{10}$ We observe a good correlation for a doping concentration of up to $20 \mathrm{~mol} \%$ but a considerable deviation for higher amounts of N-DMBI. This behavior corroborates our picture that excess dopant interrupts the nanostructure of the 
polymer, causing a considerable reduction in mobility and hence electrical conductivity at high dopant fractions. Overall, we obtain a maximum thermoelectric power factor of $0.4 \mu \mathrm{W}$ $\mathrm{K}^{-2} \mathrm{~m}^{-1}$ in the case of doping with only $10 \mathrm{~mol} \% \mathrm{~N}-\mathrm{DMBI}$, which is much higher than the highest value of $0.02 \mu \mathrm{W} \mathrm{K} \mathrm{K}^{-2}$ $\mathrm{m}^{-1}$ measured for $\mathrm{p}$ (NDI2OD-T2) (Supporting Information Figure S12c).

For p(gNDI-gT2) doped with up to $20 \mathrm{~mol} \% \mathrm{~N}-\mathrm{DMBI}$, we anticipate that the electrical conductivity is not limited by the bulk electron mobility. To gain a more complete picture of charge transport in the here-studied system, we estimate the electron mobility $\mu$ according to $\sigma=n q \mu$, where $q$ is the elementary charge, i.e., $1.6 \times 10^{-19}$ C. For a dopant concentration of $20 \mathrm{~mol} \%$, for which we have deduced the charge carrier density from EGDM as well as EPR, we obtain a value of $\mu \approx 0.2 \mathrm{~cm}^{2} \mathrm{~V}^{-1} \mathrm{~s}^{-1}$. This value is considerably higher than the electron field-effect mobility $\mu_{\mathrm{FET}} \approx 10^{-5} \mathrm{~cm}^{2} \mathrm{~V}^{-1} \mathrm{~s}^{-1}$ reported for the pristine polymer, which may arise due to the low degree of polymerization of not more than seven repeat units ${ }^{50}$ or due to the presence of polar side chains attached to the backbone of the copolymer. In contrast, the here-studied case of highly doped p(gNDI-gT2) does not appear to suffer from a low electron mobility. This observation is consistent with our recent study on p-doping of P3HT, where we likewise concluded that the molecular weight does not influence the conductivity at high dopant levels. ${ }^{3}$

Finally, we investigated the air stability of the electrical conductivity of a doped and a pristine thin film of $\mathrm{p}$ (gNDIgT2) by exposing freshly prepared samples to air while measuring the current-voltage $(I-V)$ behavior at various times. The nonlinear behavior of doped p(gNDI-gT2) samples after $30 \mathrm{~min}$ in air prevented us from extracting the electrical conductivity. Instead, we chose to plot the electrical current at $0.5 \mathrm{~V}$ (Figure 5c; cf. Supporting Information for $I-V$ curves, Figure S13). The doped and pristine samples show a markedly different response to air exposure. For the pristine polymer, we observe an immediate drop of the current. In contrast, $\mathrm{N}$ DMBI-doped p(gNDI-gT2) is able to maintain a similar current (and hence electrical conductivity) for the first $20 \mathrm{~min}$ of air exposure, which suggests that the doped polymer is more air-stable and hence can be handled outside of a protective atmosphere for at least a short period of time. However, after $30 \mathrm{~min}$ of air exposure, the current likewise drops by several orders of magnitude. After returning the samples to the glovebox, the current measured for the doped and pristine polymer quickly recovers. Subsequent annealing at $80^{\circ} \mathrm{C}$ for 10 min almost restores the current (and hence the electrical conductivity) to the initial value. We tentatively explain this behavior with adsorption of, e.g., oxygen and water from the ambient atmosphere introducing charge traps, which are subsequently desorbed from the film upon re-exposure to a protective atmosphere and annealing. ${ }^{21}$ To demonstrate the negative influence of water, we compared the conductance of the doped polymer at ambient conditions before and after placing a water droplet onto the film, which caused a 5-fold decrease in conductance (Supporting Information, Figure S14).

We have studied $\mathrm{n}$-doping of the polymer p(gNDI-gT2), which bears oligoethylene glycol-based chains, with the hydride dopant N-DMBI. The polar side chains facilitate more effective doping of the semiconducting polymer by increasing the miscibility with the dopant, resulting in a doping efficiency of $\sim 13 \%$ for a sample doped with $20 \mathrm{~mol} \% \mathrm{~N}-\mathrm{DMBI}$. We were able to prepare films with a conductivity above $10^{-1} \mathrm{~S} \mathrm{~cm}^{-1}$ and obtained a thermoelectric power factor of up to $0.4 \mu \mathrm{W} \mathrm{K} \mathrm{K}^{-2}$ $\mathrm{m}^{-1}$. Additional doping leads to segregation of the dopant, which ultimately results in a drastic reduction in the thermoelectric performance caused by a less optimal nanostructure due to excess unreacted dopant. Moreover, we found that N-DMBI-doped $\mathrm{p}$ (gNDI-gT2) displays improved air stability as compared to the pristine polymer. We conclude that polar side chains are a powerful tool for the design of more conductive and stable n-type materials.

\section{ASSOCIATED CONTENT}

\section{S Supporting Information}

The Supporting Information is available free of charge on the ACS Publications website at DOI: 10.1021/acsenergylett.7b01146.

Experimental methods; synthesis of p(gNDI-gT2); description of determination of the disorder parameter and charge carrier density; Figure S1: ${ }^{1} \mathrm{H}$ NMR spectrum of $\mathrm{p}$ (gNDI-gT2); Figure S2: MALDI ToF of $\mathrm{p}$ (gNDIgT2); Figure S3: fits of the activation energy to the charge carrier mobility; Figure S4: fits of the variabletemperature dependency; Figure S5: 3D topography images from AFM scans; Figure S6: AFM height images; Figure S7: SEM images; Figure S8: mean surface roughness plot; Figure S9: TEM images; Figure S10: $2 \mathrm{D}$ grazing-incidence wide-angle $\mathrm{X}$-ray scattering images; Figure S11: X-ray diffractograms of annealed films; Figure S12: thermoelectric properties of $p$ (NDI2ODT2) and p(gNDI-gT2); Figure S13: air stability of the I$V$ behavior; and Figure S14: influence of water droplet on the conductance (PDF)

\section{AUTHOR INFORMATION}

\section{Corresponding Author}

*E-mail: christian.muller@chalmers.se.

ORCID

Till Biskup: 0000-0003-2913-0004

L. Jan Anton Koster: 0000-0002-6558-5295

Simone Fabiano: 0000-0001-7016-6514

Christian Müller: 0000-0001-7859-7909

\section{Notes}

The authors declare no competing financial interest.

\section{ACKNOWLEDGMENTS}

We gratefully acknowledge financial support from the Swedish Research Council through Grant No. 2016-06146, the Knut and Alice Wallenberg Foundation through a Wallenberg Academy Fellowship, and the European Research Council (ERC) under Grant Agreement No. 637624. S.F. gratefully acknowledges support from VINNOVA (2015-04859) and the Swedish Research Council (2016-03979). Portions of this research were carried out at the Stanford Synchrotron Radiation Lightsource, a national user facility operated by Stanford University on behalf of the U.S. Department of Energy, Office of Basic Energy Sciences.

\section{REFERENCES}

(1) Walzer, K.; Maennig, B.; Pfeiffer, M.; Leo, K. Highly Efficient Organic Devices based on Electrically Doped Transport Layers. Chem. Rev. 2007, 107, 1233-1271.

(2) Dai, A.; Zhou, Y.; Shu, A. L.; Mohapatra, S. K.; Wang, H.; Fuentes-Hernandez, C.; Zhang, Y.; Barlow, S.; Loo, Y.-L.; Marder, S. 
R; et al. Enhanced Charge-Carrier Injection and Collection Via Lamination of Doped Polymer Layers p-Doped with a SolutionProcessible Molybdenum Complex. Adv. Funct. Mater. 2014, 24, 2197-2204.

(3) Duzhko, V. V.; Dunham, B.; Rosa, S. J.; Cole, M. D.; Paul, A.; Page, Z. A.; Dimitrakopoulos, C.; Emrick, T. N-Doped Zwitterionic Fullerenes as Interlayers in Organic and Perovskite Photovoltaic Devices. ACS Energy Lett. 2017, 2, 957-963.

(4) Pfeiffer, M.; Leo, K.; Zhou, X.; Huang, J. S.; Hofmann, M.; Werner, A.; Blochwitz-Nimoth, J. Doped Organic Semiconductors: Physics and Application in Light Emitting Diodes. Org. Electron. 2003, 4, 89-103.

(5) Zhao, X.; Zhan, X. Electron Transporting Semiconducting Polymers in Organic Electronics. Chem. Soc. Rev. 2011, 40, 37283743.

(6) Lüssem, B.; Keum, C. M.; Kasemann, D.; Naab, B.; Bao, Z.; Leo, K. Doped Organic Transistors. Chem. Rev. 2016, 116, 13714-13751.

(7) Lu, G.; Blakesley, J.; Himmelberger, S.; Pingel, P.; Frisch, J.; Lieberwirth, I.; Salzmann, I.; Oehzelt, M.; Di Pietro, R.; Salleo, A.; et al. Moderate Doping Leads to High Performance of Semiconductor/Insulator Polymer Blend Transistors. Nat. Commun. 2013, 4, 1588

(8) Wei, P.; Oh, J. H.; Dong, G.; Bao, Z. Use of a 1H-Benzoimidazole Derivative as an $\mathrm{n}$-Type Dopant and to Enable Air-Stable SolutionProcessed n-Channel Organic Thin-Film Transistors. J. Am. Chem. Soc. 2010, 132, 8852-8853.

(9) Russ, B.; Glaudell, A.; Urban, J. J.; Chabinyc, M. L.; Segalman, R. A. Organic Thermoelectric Materials for Energy Harvesting and Temperature Control. Nat. Rev. Mater. 2016, 1, 16050.

(10) Glaudell, A. M.; Cochran, J. E.; Patel, S. N.; Chabinyc, M. L. Impact of the Doping Method on Conductivity and Thermopower in Semiconducting Polythiophenes. Adv. Energy Mater. 2015, 5, 1401072.

(11) Kroon, R.; Mengistie, D. A.; Kiefer, D.; Hynynen, J.; Ryan, J. D.; Yu, L.; Müller, C. Thermoelectric plastics: from design to synthesis, processing and structure-property relationships. Chem. Soc. Rev. 2016, 45, 6147-6164.

(12) Duong, D. T.; Wang, C.; Antono, E.; Toney, M. F.; Salleo, A. The Chemical and Structural Origin of Efficient p-Type Doping in P3HT. Org. Electron. 2013, 14, 1330-1336.

(13) Kroon, R.; Kiefer, D.; Stegerer, D.; Yu, L.; Sommer, M.; Müller, C. Polar Side Chains Enhance Processability, Electrical Conductivity, and Thermal Stability of a Molecularly p-Doped Polythiophene. Adv. Mater. 2017, 29, 1700930.

(14) Schlitz, R. A.; Brunetti, F. G.; Glaudell, A. M.; Miller, P. L.; Brady, M. A.; Takacs, C. J.; Hawker, C. J.; Chabinyc, M. L. SolubilityLimited Extrinsic n-Type Doping of a High Electron Mobility Polymer for Thermoelectric Applications. Adv. Mater. 2014, 26, 2825-2830.

(15) Euvrard, J.; Revaux, A.; Bayle, P.-A.; Bardet, M.; Vuillaume, D.; Kahn, A. The Formation of Polymer-Dopant Aggregates as a Possible Origin of Limited Doping Efficiency at High Dopant Concentration. Org. Electron. 2018, 53, 135-140.

(16) Salzmann, I.; Heimel, G.; Oehzelt, M.; Winkler, S.; Koch, N. Molecular Electrical Doping of Organic Semiconductors: Fundamental Mechanisms and Emerging Dopant Design Rules. Acc. Chem. Res. 2016, 49, 370-378.

(17) Jacobs, I. E.; Moule, A. J. Controlling Molecular Doping in Organic Semiconductors. Adv. Mater. 2017, 29, 1703063.

(18) Patel, S. N.; Glaudell, A. M.; Kiefer, D.; Chabinyc, M. L. Increasing the Thermoelectric Power Factor of a Semiconducting Polymer by Doping from the Vapor Phase. ACS Macro Lett. 2016, 5, 268-272.

(19) Patel, S. N.; Glaudell, A. M.; Peterson, K. A.; Thomas, E. M.; O'Hara, K. A.; Lim, E.; Chabinyc, M. L. Morphology Controls the Thermoelectric Power Factor of a Doped Semiconducting Polymer. Sci. Adv. 2017, 3, e1700434.

(20) Li, H.; DeCoster, M. E.; Ireland, R. M.; Song, J.; Hopkins, P. E.; Katz, H. E. Modification of the Poly(bisdodecylquaterthiophene) Structure for High and Predominantly Nonionic Conductivity with Matched Dopants. J. Am. Chem. Soc. 2017, 139, 11149-11157.
(21) de Leeuw, D. M.; Simenon, M. M. J.; Brown, A. R.; Einerhand, R. E. F. Stability of n-Type Doped Conducting Polymers and Consequences for Polymeric Microelectronic Devices. Synth. Met. 1997, 87, 53-59.

(22) Cho, N.; Yip, H.-L.; Davies, J. A.; Kazarinoff, P. D.; Zeigler, D. F.; Durban, M. M.; Segawa, Y.; O’Malley, K. M.; Luscombe, C. K.; Jen, A. K. Y. In-situ Crosslinking and n-Doping of Semiconducting Polymers and Their Application as Efficient Electron-Transporting Materials in Inverted Polymer Solar Cells. Adv. Energy Mater. 2011, 1, $1148-1153$.

(23) Higgins, A.; Mohapatra, S. K.; Barlow, S.; Marder, S. R.; Kahn, A. Dopant Controlled Trap-Filling and Conductivity Enhancement in an Electron-Transport Polymer. Appl. Phys. Lett. 2015, 106, 163301.

(24) Liang, Y.; Chen, Z.; Jing, Y.; Rong, Y.; Facchetti, A.; Yao, Y. Heavily n-Dopable $\pi$-Conjugated Redox Polymers with Ultrafast Energy Storage Capability. J. Am. Chem. Soc. 2015, 137, 4956-4959.

(25) Madan, D.; Zhao, X.; Ireland, R. M.; Xiao, D.; Katz, H. E. Conductivity and Power Factor Enhancement of n-Type Semiconducting Polymers using Sodium Silica Gel Dopant. APL Mater. 2017, 5, 086106.

(26) Naab, B. D.; Gu, X.; Kurosawa, T.; To, J. W. F.; Salleo, A.; Bao, Z. Role of Polymer Structure on the Conductivity of N-Doped Polymers. Adv. Electron. Mater. 2016, 2, 1600004.

(27) Naab, B. D.; Guo, S.; Olthof, S.; Evans, E. G.; Wei, P.; Millhauser, G. L.; Kahn, A.; Barlow, S.; Marder, S. R.; Bao, Z. Mechanistic Study on the Solution-Phase n-Doping of 1,3-Dimethyl-2Aryl-2,3-Dihydro-1H-Benzoimidazole Derivatives. J. Am. Chem. Soc. 2013, 135, 15018-15025.

(28) Wang, S.; Sun, H.; Ail, U.; Vagin, M.; Persson, P. O.; Andreasen, J. W.; Thiel, W.; Berggren, M.; Crispin, X.; Fazzi, D.; et al. Thermoelectric Properties of Solution-Processed n-Doped LadderType Conducting Polymers. Adv. Mater. 2016, 28, 10764-10771.

(29) Zhang, Y.; Phan, H.; Zhou, H.; Zhang, X.; Zhou, J.; Moudgil, K.; Barlow, S.; Marder, S. R.; Facchetti, A.; Nguyen, T.-Q. Electron Transport and Nanomorphology in Solution-Processed Polymeric Semiconductor n-Doped with an Air-Stable Organometallic Dimer. Adv. Electron. Mater. 2017, 3, 1600546.

(30) Ma, W.; Shi, K.; Wu, Y.; Lu, Z. Y.; Liu, H. Y.; Wang, J. Y.; Pei, J. Enhanced Molecular Packing of a Conjugated Polymer with High Organic Thermoelectric Power Factor. ACS Appl. Mater. Interfaces 2016, 8, 24737-24743.

(31) Shi, K.; Zhang, F.; Di, C. A.; Yan, T. W.; Zou, Y.; Zhou, X.; Zhu, D.; Wang, J. Y.; Pei, J. Toward High Performance n-Type Thermoelectric Materials by Rational Modification of BDPPV Backbones. J. Am. Chem. Soc. 2015, 137, 6979-6982.

(32) Zhao, X.; Madan, D.; Cheng, Y.; Zhou, J.; Li, H.; Thon, S. M.; Bragg, A. E.; DeCoster, M. E.; Hopkins, P. E.; Katz, H. E. High Conductivity and Electron-Transfer Validation in an n-Type FluorideAnion-Doped Polymer for Thermoelectrics in Air. Adv. Mater. 2017, 29, 1606928.

(33) Cho, N.; Yip, H.-L.; Hau, S. K.; Chen, K.-S.; Kim, T.-W.; Davies, J. A.; Zeigler, D. F.; Jen, A. K. Y. n-Doping of Thermally Polymerizable Fullerenes as an Electron Transporting Layer for Inverted Polymer Solar Cells. J. Mater. Chem. 2011, 21, 6956.

(34) Gao, F.; Liu, Y.; Xiong, Y.; Wu, P.; Hu, B.; Xu, L. Fabricate Organic Thermoelectric Modules use Modified PCBM and PEDOT: PSS Materials. Front. Optoelectron. 2017, 10, 117-123.

(35) Harada, K.; Li, F.; Maennig, B.; Pfeiffer, M.; Leo, K. Ionized Impurity Scattering in n-Doped C60 Thin Films. Appl. Phys. Lett. 2007, 91, 092118.

(36) Li, C. Z.; Chueh, C. C.; Ding, F.; Yip, H. L.; Liang, P. W.; Li, X.; Jen, A. K. Doping of Fullerenes via Anion-Induced Electron Transfer and its Implication for Surfactant Facilitated High Performance Polymer Solar Cells. Adv. Mater. 2013, 25, 4425-4430.

(37) Li, F.; Pfeiffer, M.; Werner, A.; Harada, K.; Leo, K.; Hayashi, N.; Seki, K.; Liu, X.; Dang, X.-D. Acridine Orange Base as a Dopant for nDoping of C60 Thin Films. J. Appl. Phys. 2006, 100, 023716. 
(38) Li, F.; Werner, A.; Pfeiffer, M.; Leo, K.; Liu, X. Leuco Crystal Violet as a Dopant for $\mathrm{n}$-Doping of Organic Thin Films of Fullerene C60. J. Phys. Chem. B 2004, 108, 17076-17082.

(39) Liu, J.; Qiu, L.; Portale, G.; Koopmans, M.; Ten Brink, G.; Hummelen, J. C.; Koster, L. J. A. N-Type Organic Thermoelectrics: Improved Power Factor by Tailoring Host-Dopant Miscibility. Adv. Mater. 2017, 29, 1701641.

(40) Menke, T.; Ray, D.; Meiss, J.; Leo, K.; Riede, M. In-Situ Conductivity and Seebeck Measurements of Highly Efficient nDopants in Fullerene C60. Appl. Phys. Lett. 2012, 100, 093304.

(41) Menke, T.; Wei, P.; Ray, D.; Kleemann, H.; Naab, B. D.; Bao, Z.; Leo, K.; Riede, M. A Comparison of Two Air-Stable Molecular nDopants for C60. Org. Electron. 2012, 13, 3319-3325.

(42) Naab, B. D.; Zhang, S.; Vandewal, K.; Salleo, A.; Barlow, S.; Marder, S. R.; Bao, Z. Effective Solution- and Vacuum-Processed nDoping by Dimers of Benzimidazoline Radicals. Adv. Mater. 2014, 26, 4268-4272.

(43) Qiu, L.; Liu, J.; Alessandri, R.; Qiu, X.; Koopmans, M.; Havenith, R. W. A.; Marrink, S. J.; Chiechi, R. C.; Anton Koster, L. J.; Hummelen, J. C. Enhancing Doping Efficiency by Improving HostDopant Miscibility for Fullerene-Based n-Type Thermoelectrics. J. Mater. Chem. A 2017, 5, 21234-21241.

(44) Tietze, M. L.; Rose, B. D.; Schwarze, M.; Fischer, A.; Runge, S.; Blochwitz-Nimoth, J.; Lüssem, B.; Leo, K.; Brédas, J.-L. Passivation of Molecular n-Doping: Exploring the Limits of Air Stability. Adv. Funct. Mater. 2016, 26, 3730-3737.

(45) Wei, P.; Menke, T.; Naab, B. D.; Leo, K.; Riede, M.; Bao, Z. 2(2-Methoxyphenyl)-1,3-dimethyl-1H-benzoimidazol-3-ium Iodide as a New Air-Stable n-Type Dopant for Vacuum-Processed Organic Semiconductor Thin Films. J. Am. Chem. Soc. 2012, 134, 3999-4002.

(46) Chen, Z.; Zheng, Y.; Yan, H.; Facchetti, A. Naphthalenedicarboximide- vs Perylenedicarboximide-based Copolymers. Synthesis and Cemiconducting Properties in Bottom-Gate N-Channel Organic Transistors. J. Am. Chem. Soc. 2009, 131, 8-9.

(47) Wang, Y.; Nakano, M.; Michinobu, T.; Kiyota, Y.; Mori, T.; Takimiya, K. Naphthodithiophenediimide-Benzobisthiadiazole-Based Polymers: Versatile n-Type Materials for Field-Effect Transistors and Thermoelectric Devices. Macromolecules 2017, 50, 857-864.

(48) Perry, E. E.; Chiu, C.-Y.; Moudgil, K.; Schlitz, R. A.; Takacs, C. J.; O’Hara, K. A.; Labram, J. G.; Glaudell, A. M.; Sherman, J. B.; Barlow, S.; et al. High Conductivity in a Nonplanar n-Doped Ambipolar Semiconducting Polymer. Chem. Mater. 2017, 29, 97429750.

(49) Li, J.; Rochester, C. W.; Jacobs, I. E.; Aasen, E. W.; Friedrich, S.; Stroeve, P.; Moulé, A. J. The Effect of Thermal Annealing on Dopant Site Choice in Conjugated Polymers. Org. Electron. 2016, 33, 23-31.

(50) Giovannitti, A.; Nielsen, C. B.; Sbircea, D. T.; Inal, S.; Donahue, M.; Niazi, M. R.; Hanifi, D. A.; Amassian, A.; Malliaras, G. G.; Rivnay, J.; et al. N-type Organic Electrochemical Transistors with Stability in Water. Nat. Commun. 2016, 7, 13066.

(51) Nielsen, C. B.; Giovannitti, A.; Sbircea, D. T.; Bandiello, E.; Niazi, M. R.; Hanifi, D. A.; Sessolo, M.; Amassian, A.; Malliaras, G. G.; Rivnay, J.; et al. Molecular Design of Semiconducting Polymers for High-Performance Organic Electrochemical Transistors. J. Am. Chem. Soc. 2016, 138, 10252-10259.

(52) Giovannitti, A.; Sbircea, D. T.; Inal, S.; Nielsen, C. B.; Bandiello, E.; Hanifi, D. A.; Sessolo, M.; Malliaras, G. G.; McCulloch, I.; Rivnay, J. Controlling the Mode of Operation of Organic Transistors through Side-Chain Engineering. Proc. Natl. Acad. Sci. U. S. A. 2016, 113, 12017-12022.

(53) Colter, A. K.; Saito, G.; Sharom, F. J. Hydride Transfer Reactions. Oxidation of N-Methylacridan by $\pi$ Acceptors. Can. J. Chem. 1977, 55, 2741-2751.

(54) Colter, A. K.; Saito, G.; Sharom, F. J.; Hong, A. P. Hydride Transfer Reactions. Oxidation of N-Methylacridan by 1,4-Benzoquinone and Related $\pi$ Acceptors. J. Am. Chem. Soc. 1976, 98, 78337835.

(55) Guo, X.; Watson, M. D. Conjugated Polymers from Naphthalene Bisimide. Org. Lett. 2008, 10, 5333-5336.
(56) Pasveer, W. F.; Cottaar, J.; Tanase, C.; Coehoorn, R.; Bobbert, P. A.; Blom, P. W.; de Leeuw, D. M.; Michels, M. A. Unified Description of Charge-Carrier Mobilities in Disordered Semiconducting Polymers. Phys. Rev. Lett. 2005, 94, 206601.

(57) Craciun, N. I.; Wildeman, J.; Blom, P. W. Universal Arrhenius Temperature Activated Charge Transport in Diodes from Disordered Organic Semiconductors. Phys. Rev. Lett. 2008, 100, 056601-056604.

(58) Martens, H. C. F.; Blom, P. W. M.; Schoo, H. F. M. Comparative Study of Hole Transport in Poly(p-phenylene vinylene) Derivatives. Phys. Rev. B: Condens. Matter Mater. Phys. 2000, 61, 74897493.

(59) Rivnay, J.; Toney, M. F.; Zheng, Y.; Kauvar, I. V.; Chen, Z.; Wagner, V.; Facchetti, A.; Salleo, A. Unconventional Face-on Texture and Exceptional In-Plane Order of a High Mobility n-Type Polymer. Adv. Mater. 2010, 22, 4359-4363.

(60) Schuettfort, T.; Huettner, S.; Lilliu, S.; Macdonald, J. E.; Thomsen, L.; McNeill, C. R. Surface and Bulk Structural Characterization of a High-Mobility Electron-Transporting Polymer. Macromolecules 2011, 44, 1530-1539.

(61) Brinkmann, M.; Gonthier, E.; Bogen, S.; Tremel, K.; Ludwigs, S.; Hufnagel, M.; Sommer, M. Segregated versus mixed interchain stacking in highly oriented films of naphthalene diimide bithiophene copolymers. ACS Nano 2012, 6, 10319-10326.

(62) Takacs, C. J.; Treat, N. D.; Kramer, S.; Chen, Z.; Facchetti, A.; Chabinyc, M. L.; Heeger, A. J. Remarkable Order of a HighPerformance Polymer. Nano Lett. 2013, 13, 2522-2527.

(63) Hynynen, J.; Kiefer, D.; Yu, L.; Kroon, R.; Munir, R.; Amassian, A.; Kemerink, M.; Müller, C. Enhanced Electrical Conductivity of Molecularly p-Doped Poly(3-hexylthiophene) through Understanding the Correlation with Solid-State Order. Macromolecules 2017, 50, $8140-8148$ 